\title{
Un raro caso di ectopia renale
}

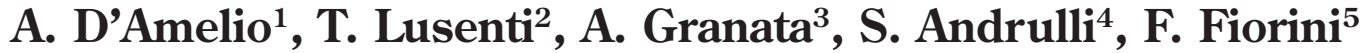

\author{
${ }^{1}$ Ospedale "V. Fazzi", Lecce \\ ${ }^{2}$ Ospedale "S. Maria Nuova”, Reggio Emilia \\ ${ }^{3}$ Ospedale "V. Emanuele”, Catania \\ ${ }^{4}$ Ospedale “A. Manzoni”, Lecco \\ ${ }^{5}$ Ospedale “G. Borea”, Sanremo (Imperia) \\ per il Gruppo di Studio di Ecografia Renale della Società Italiana di Nefrologia
}

\section{Presentazione del caso}

Un maschio di 46 anni giunge all'osservazione dell'ambulatorio di Ecografia Nefrologica per riferita colica reno-ureterale destra.

In anamnesi familiare non è riferita la presenza di malattie renali, nefrolitiasi, diabete mellito e ipertensione arteriosa.

L'anamnesi remota rivela: non pregressi episodi di colica renale e buona salute riferita fino all'età di 24 anni (1981) quando, in corso di episodio bronchitico viene sottoposto a Rx torace che pone diagnosi di "cisti polmonare sinistra di ndd". Il Paziente viene ricoverato in ambito internistico dove effettua una toracotomia esplorativa che esclude la presenza del problema cistico. Da allora il Paziente (non fumatore) riferisce di accusare almeno un episodio bronchitico di lieve entità all'anno. All'età di 33 anni (1990) il Paziente presenta un focolaio broncopneumonico specifico, trattato con terapia adeguata. Sono segnalati inoltre sporadici episodi di tachicardia e saltuarie salve di extrasistoli.

I principali esami di laboratorio (esame urine, urinocoltura, VES, emocromo, glicemia, uricemia, funzionalità renale ed epatica, elettroliti sierici ed urinari) sono tutti nella norma.

L'esame ecografico renale dimostra rene destro in sede, a contorni regolari, di normale morfologia e dimensioni, con assenza di dilatazioni calicopieliche e/o litiasi. Il rene sinistro non è visualizzabile nella sua normale sede e la loggia renale sinistra risulta disabitata: in realtà dopo attento esame, il rene, normale per morfologia e dimensioni, appare esaminabile in sede ectopica, con scansioni intercostali, anterolaterali e posteriori nella parte basale sinistra della cavità toracica, in posizione postero-superiore rispetto alla milza (Figg. 1, 2).

L'Rx torace, eseguito immediatamente dopo, dimostra a livello della cupola diaframmatica sinistra la presenza di una zona iperdiafana ovalare (Fig. 3).

La TC toracica conferma la presenza renale a livello polmonare inferiore sinistro (Fig. 4), mentre la Angio RM descrive una relaxatio diaframmatica posteriore sinistra (nelle slices anteriori il diaframma appare normoposizionato) con conseguente risalita del rene omolaterale con il grasso perirenale: normale l'origine dall'aorta addominale dell'arteria renale sinistra che risale ad "uncino" ed assume decorso verticale fino alla pelvi renale corrispondente. Non alterazioni a carico degli ureteri con presenza di pelvi bifida bilateralmente (Figg. 5, 6).

\section{Conclusione}

La relaxatio diaframmatica è una patologia del diaframma che non deve essere confusa con le ernie diaframmatiche "vere" (Fig. 7) in quanto, come nel nostro caso, non vi è la presenza di un orifizio anomalo del diaframma ma di un suo "cedimento parziale", senza che vi sia interruzione della sua struttura (1): si tratta di un abnorme sopraelevamento di un emidiaframma generalmente di natura paralitica. Il termine relaxatio è frequentemente usato indifferentemente con il termine eventratio: in realtà, mentre nella "eventratio" la paralisi è congenita, per processi patologici prenatali oppure per processi traumatici da parto coinvolgenti il nervo frenico, la relaxatio 


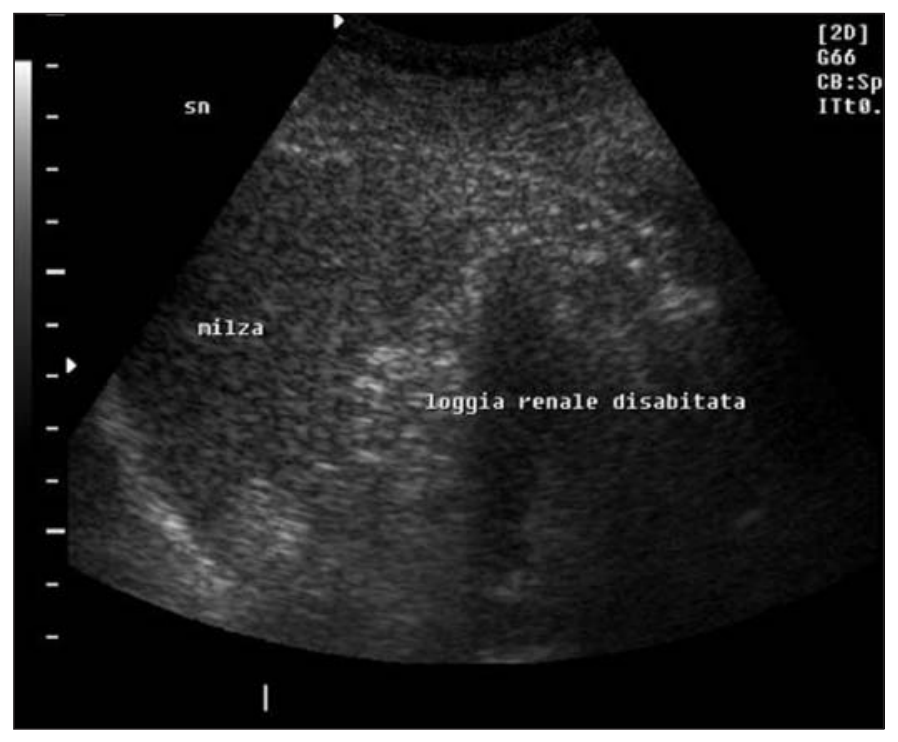

Fig. 1 - La normale scansione ecografica sottosplenica longitudinale obliqua posteriore visualizza la loggia renale sinistra disabitata.

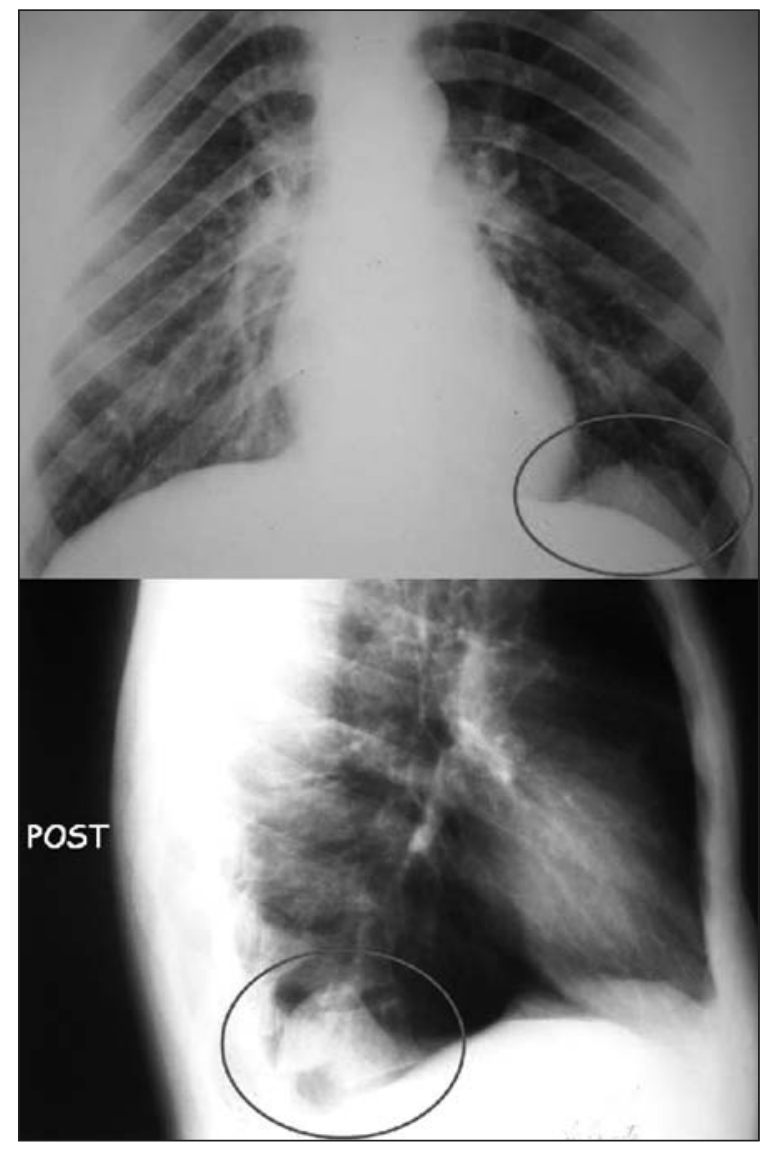

Fig. 3 - L'Rx torace visualizza in entrambe le proiezioni la presenza di un'area iperdiafana grossolanamente ovalare, a livello della base polmonare sinistra.

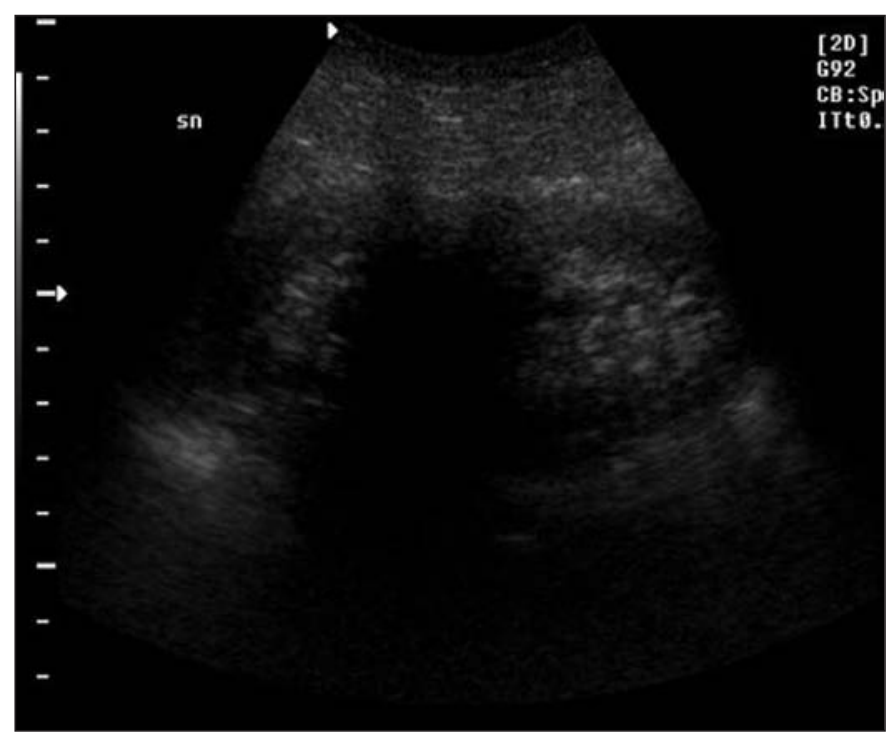

Fig. 2 - La scansione intercostale posteriore sovra-splenica dimostra la presenza di un rene di normale morfologia e dimensioni (pur in presenza di coni d'ombra costali).

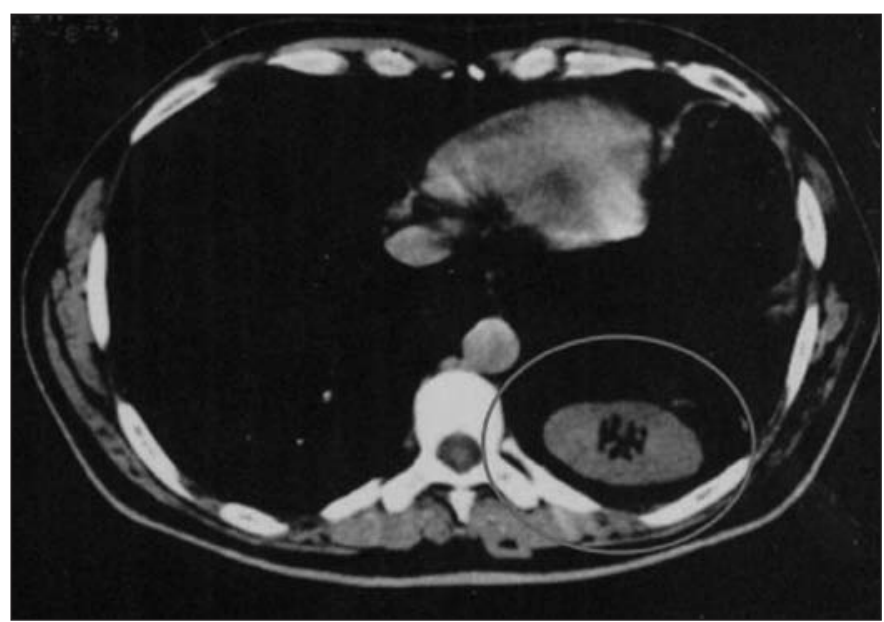

Fig. 4 - Alla TC è chiara la presenza di un rene a livello della base polmonare sinistra.

è una paralisi postnatale, che consegue a frenico-exeresi e a lesioni traumatiche,infiammatorie o neoplastiche del nervo frenico: una causa non paralitica di relaxatio è la miosite atrofizzante del diaframma (2-4).

Il quadro clinico e la sintomatologia della relaxatio diaframmatica risultano proporzionali all'ampiezza e alla sede: nelle forme più limitate saranno evidenziabili disturbi polmonari con bronchiti croniche recidivanti (analogamente a quanto riportato nel nostro caso) fino 

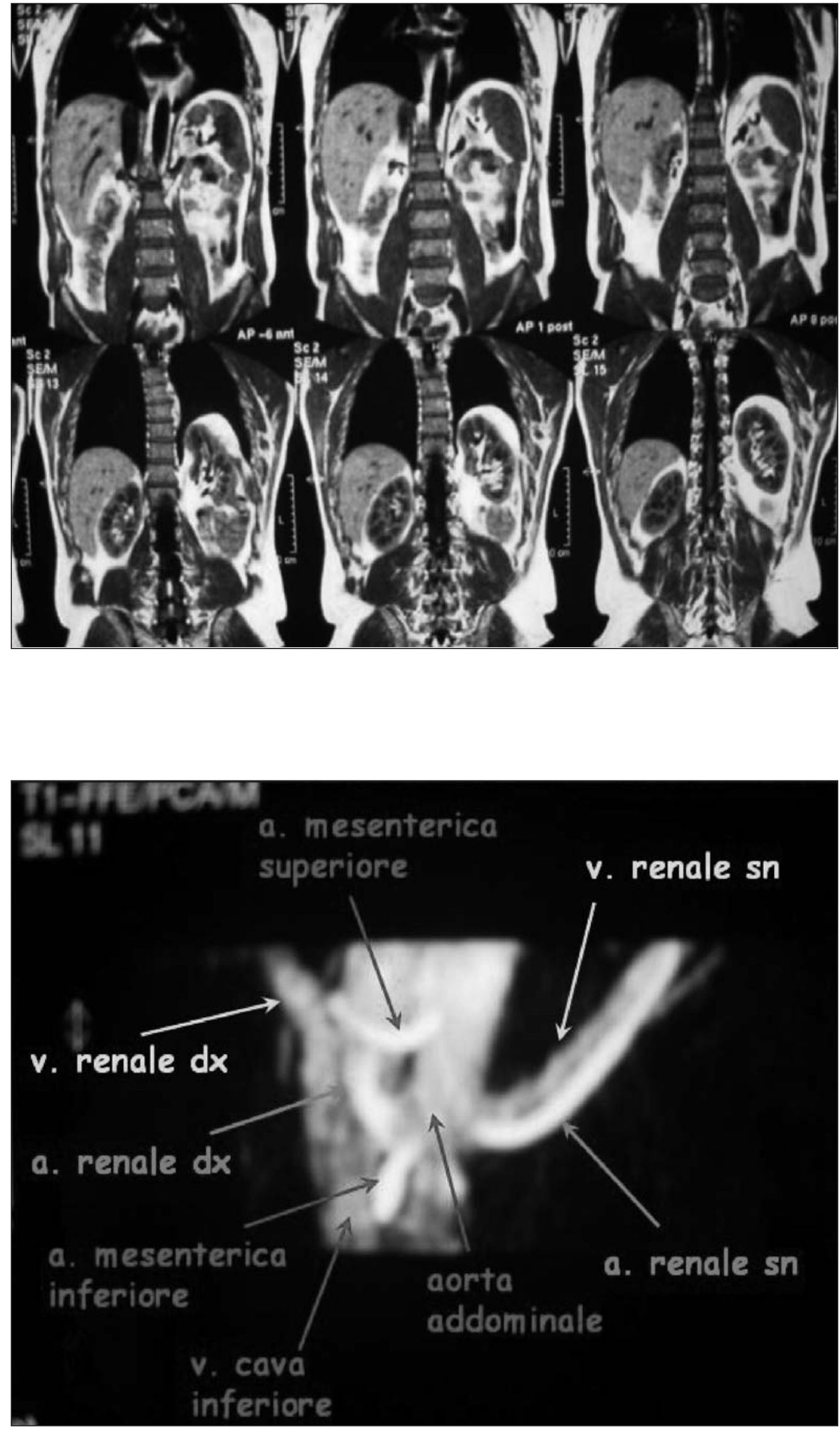

Fig. 5 - L'angioRM dimostra chiaramente come la relaxatio diaframmatica non sia presente a livello della parte anteriore del diaframma sinistro (sequenza in alto) e come invece con le scansioni posteriori (sequenza in basso) sia evidente come la cupola diaframmatica venga spinta in alto dalla comparsa, supero-posteriormente alla milza, del rene omolaterale.

Fig. 6 - L'angioRM evidenzia un'origine normale delle arterie renali, con una direzione cranio-laterale di quella sinistra. 
malia di posizione del viscere, ha permesso un'ottima valutazione della morfologia e dell'ecostruttura e, integrandosi con metodiche strumentali radiologiche, ha permesso una valutazione completa e globale dell'anomalia riscontrata.

Indirizzo degli Autori:

Fulvio Fiorini, MD

UO Nefrologia e Dialisi

Ospedale "G. Borea"

Via Borea 56

18038 Sanremo (IM)

f.fiorini@asl1.liguria.it

\section{Bibliografia}

1. Vazquez AF, Vicente Prados FJ, et al. Intrathoracic ectopic kidney in a adult: case report and bibliographic review. Arch Esp Urol 2008; 61(5): 631-3.

2. Pfister-Goedeke L, Brunier E. Intrathoracic kidney in childhood with special reference to secondary renal transport in Bochdalek's hernia. Helv Paediatr Acta 1979; 34(4): 345-57.

Fig. 7 - Sono schematicamente riportate: A) ernia em brionale; B) ernia fetale; C) eventrazione parziale; D) eventrazione totale.

a turbe dell'attività cardiaca (presenti in modo sfumato nel caso clinico in oggetto) e della sfera digestiva (1). Anche in questo caso, l'ecografia ha costituito l'esame diagnostico di base nello studio renale: è stata in grado infatti di individuare immediatamente l'ano-

3. Subramanian VS, Goldfarb DA. Right-sided intrathoracic renal ectopia with Bochdalek's hernia. Urology 2008; 72(5): 1016-7.

4. Dingeldein MW, Kane D, Kim AW, et al. Bilateral intrathoracic kidneys and adrenal glands associated with posterior congenital diaphragmatic hernias. Ann Thor Surg 2008; 86(2): 651-4. 